\title{
On Classification of Real Hypersurfaces in a Complex Space Form with $\eta$-recurrent Shape Operator
}

\author{
Tee-How LOO \\ University of Malaya \\ (Communicated by K. Matsuzaki)
}

\begin{abstract}
In this paper, we classify real hypersurfaces in a non-flat complex space with $\eta$-recurrent shape operator.
\end{abstract}

\section{Introduction}

Let $M_{n}(c)$ be an $n$-dimensional complete and simply connected non-flat complex space form with complex structure $J$ of constant holomorphic sectional curvature $4 c$, i.e., it is either a complex projective space $\mathbf{C} P_{n}$ (for $c>0$ ), or a complex hyperbolic space $\mathbf{C} H_{n}$ (for $c<0$ ).

Suppose $M$ is a connected real hypersurface in $M_{n}(c)$ and $N$ is a unit normal vector field of $M$. We denote by $\Gamma(\mathcal{V})$ the module of all differentiable sections on the vector bundle $\mathcal{V}$ over $M$. Let $\xi=-J N$ be the structure vector field and $A$ the shape operator on $M$. A Hopf hypersurface $M$ in $M_{n}(c)$ is characterized by the condition that the structure vector field $\xi$ is principal, i.e., $A \xi=\alpha \xi$, and it can be shown that this principal curvature $\alpha$ is a constant.

Typical examples of Hopf hypersurfaces are those with constant principal curvatures, nowadays, so-called real hypersurfaces of type $A_{1}, A_{2}, B, C, D$ and $E$ (resp. of type $A_{0}$, $A_{1}, A_{2}$ and $B$ ) in $\mathbf{C} P_{n}$ (resp. in $\mathbf{C} H_{n}$ ) (cf. [14], [12]). These real hypersurfaces can be expressed as tubes of constant radius over certain holomorphic or totally real submanifolds, and a self-tube in the ambient space (cf. [1], [2], [5]).

Other than these Hopf hypersurfaces, another example of real hypersurfaces in $M_{n}(c)$ are the class of ruled real hypersurfaces. Ruled real hypersurfaces in $M_{n}(c)$ are characterized by having a one-codimensional foliation whose leaves are complex totally geodesic hyperplanes in $M_{n}(c)$. The geometry of ruled real hypersurfaces in $M_{n}(c)$ was studied in [10].

The study of real hypersurfaces in a non-flat complex space form has been an active field in the past few decades. One of the first results is the non-existence of real hypersurfaces with parallel shape operator $A$, i.e., $\nabla A=0$, where $\nabla$ is the Levi-Civita connection of $M$.

Received September 17, 2013; revised February 28, 2014

2010 Mathematics Subject Classification: 53B25 (Primary), 53C15 (Secondary)

Key words and phrases: complex space form, Hopf hypersurfaces, ruled real hypersurfaces, $\eta$-recurrent shape operator

This work was supported in part by the UMRG research grant (Grant No. RG163/11AFR). 
This fact is an immediate consequence of the Codazzi equation of such a real hypersurface. Motivated by this, Kimura and Maeda [6] studied the weaker notion of $\eta$-parallelism. The shape operator $A$ is said to be $\eta$-parallel if it satisfies the following condition:

$$
\left\langle\left(\nabla_{X} A\right) Y, Z\right\rangle=0
$$

for any $X, Y$ and $Z \in \Gamma(\mathcal{D})$, where $\mathcal{D}:=\operatorname{Span}\{\xi\}^{\perp}$, called the (maximal) holomorphic distribution on $M$. A number of results concerning real hypersurfaces with $\eta$-parallel shape operator have been obtained (cf. [6], [7], [8], [13]). In particular, a complete classification of real hypersurfaces in $M_{n}(c)$ with $\eta$-parallel shape operator was proved in [8] (cf. Theorem 4).

In another way to weaker the parallelism, Hamada [3] studied the recurrence of the shape operator of real hypersurfaces in $\mathbf{C} P_{n}$. The shape operator $A$ is said to be recurrent if $\nabla A=$ $A \otimes \omega$ for some 1-form $\omega$ in $M$. It was showed in [3] that the recurrence is also too strong to be satisfied by the shape operator of real hypersurfaces in $\mathbf{C} P_{n}$.

The shape operator $A$ is said to be $\eta$-recurrent if there is a 1-form $\omega$ on $M$ such that

$$
\left\langle\left(\nabla_{X} A\right) Y, Z\right\rangle=\omega(X)\langle A X, Y\rangle
$$

for any $X, Y, Z \in \Gamma(\mathcal{D})$. The $\eta$-parallelism and recurrence can be considered as special cases of $\eta$-recurrence. Hopf hypersurfaces in $M_{n}(c)$ with $\eta$-recurrent shape operator were classified in $[4,11]$.

THEOREM 1 ([4, 11]). Let $M$ be a Hopf hypersurface in $M_{n}(c), n \geq 3, c \neq 0$. Then the shape operator $A$ is $\eta$-recurrent if and only if $M$ is locally congruent to one of the following spaces:

(a) For $c>0$ :

$\left(A_{1}\right)$ a tube over hyperplane $\mathbf{C} P_{n-1}$;

$\left(A_{2}\right)$ a tube over totally geodesic $\mathbf{C} P_{k}$, where $1 \leq k \leq n-2$;

(B) a tube over complex quadric $Q_{n-1}$.

(b) For $c<0$ :

$\left(A_{0}\right)$ a horosphere;

$\left(A_{1}\right)$ a geodesic hypersphere or a tube over hyperplane $\mathbf{C} H_{n-1}$;

$\left(A_{2}\right)$ a tube over totally geodesic $\mathbf{C} H_{k}$, where $1 \leq k \leq n-2$;

(B) a tube over totally real hyperbolic space $\mathbf{R} H^{n}$.

The purpose of this paper is to improve the above theorem and classify real hypersurfaces in $M_{n}(c)$ with $\eta$-recurrent shape operator, i.e., we prove the following theorem.

THEOREM 2. Let $M$ be a real hypersurface in $M_{n}(c), n \geq 3, c \neq 0$. Then its shape operator $A$ is $\eta$-recurrent if and only if $M$ is locally congruent to to a ruled real hypersurface or one of the following spaces:

(a) For $c>0$ :

$\left(A_{1}\right)$ a tube over hyperplane $\mathbf{C} P_{n-1}$;

$\left(A_{2}\right)$ a tube over totally geodesic $\mathbf{C} P_{k}$, where $1 \leq k \leq n-2$; 
(B) a tube over complex quadric $Q_{n-1}$.

(b) For $c<0$ :

$\left(A_{0}\right)$ a horosphere;

$\left(A_{1}\right)$ a geodesic hypersphere or a tube over hyperplane $\mathbf{C} H_{n-1}$;

$\left(A_{2}\right)$ a tube over totally geodesic $\mathbf{C} H_{k}$, where $1 \leq k \leq n-2$;

(B) a tube over totally real hyperbolic space $\mathbf{R} H^{n}$.

\section{Preliminaries}

In this section we shall recall some fundamental identities and known results in the theory of real hypersurfaces in a complex space form and fix some notations.

Let $M$ be a connected real hypersurface isometrically immersed in $M_{n}(c), n \geq 3, N$ a unit normal vector field on $M$ and $\langle$,$\rangle the Riemannian metric on M$. We define a tensor field $\phi$ of type $(1,1)$, a vector field $\xi$ and a 1 -form $\eta$ by

$$
J X=\phi X+\eta(X) N, \quad J N=-\xi, \quad \eta(X)=\langle\xi, X\rangle
$$

for any $X \in \Gamma(T M)$. Then we have

$$
\phi^{2} X=-X+\eta(X) \xi, \quad \phi \xi=0, \quad \eta(\phi X)=0, \quad \eta(\xi)=1 .
$$

Denote by $\nabla$ the Levi-Civita connection and $A$ the shape operator on $M$. Then

$$
\left(\nabla_{X} \phi\right) Y=\eta(Y) A X-\langle A X, Y\rangle \xi, \quad \nabla_{X} \xi=\phi A X
$$

for any $X, Y \in \Gamma(T M)$.

Let $R$ be the curvature tensor of $M$. Then the equations of Gauss and Codazzi are given respectively by

$$
\begin{array}{r}
R(X, Y) Z=c\{\langle Y, Z\rangle X-\langle X, Z\rangle Y+\langle\phi Y, Z\rangle \phi X-\langle\phi X, Z\rangle \phi Y \\
-2\langle\phi X, Y\rangle \phi Z\}+\langle A Y, Z\rangle A X-\langle A X, Z\rangle A Y \\
\left(\nabla_{X} A\right) Y-\left(\nabla_{Y} A\right) X=c\{\eta(X) \phi Y-\eta(Y) \phi X-2\langle\phi X, Y\rangle \xi\} .
\end{array}
$$

This following lemma is needed in the next section.

LEMMA 3 ([9]). Let $M$ be a non-Hopf real hypersurface in $M_{n}(c), n \geq 3, c \neq 0$. Suppose $A \xi=\alpha \xi+\beta U$ and $A U=\beta \xi+\gamma U$, where $\beta=\|\phi A \xi\|(>0)$ and $U=$ $-\beta^{-1} \phi^{2} A \xi$. If there exists a unit vector field $Z \perp \xi, U, \phi U$ such that $A Z=\lambda Z$ and $A \phi Z=$ $\lambda \phi Z$, then

$$
(\lambda-\gamma)\left(\lambda^{2}-\alpha \lambda-c\right)-\beta^{2} \lambda=0 .
$$

Finally, we state without proof the following result concerning real hypersurfaces in $M_{n}(c)$ with $\eta$-parallel shape operator. 
THEOREM 4 ([8]). Let $M$ be a real hypersurface in $M_{n}(c), n \geq 3, c \neq 0$. Then its shape operator $A$ is $\eta$-parallel if and only if $M$ is locally congruent to a ruled real hypersurface or one of the following spaces:

(a) For $c>0$ :

$\left(A_{1}\right)$ a tube over hyperplane $\mathbf{C} P_{n-1}$;

$\left(A_{2}\right)$ a tube over totally geodesic $\mathbf{C} P_{k}$, where $1 \leq k \leq n-2$;

(B) a tube over complex quadric $Q_{n-1}$.

(b) For $c<0$ :

$\left(A_{0}\right)$ a horosphere;

$\left(A_{1}\right)$ a geodesic hypersphere or a tube over hyperplane $\mathbf{C} H_{n-1}$;

$\left(A_{2}\right)$ a tube over totally geodesic $\mathbf{C} H_{k}$, where $1 \leq k \leq n-2$;

(B) a tube over totally real hyperbolic space $\mathbf{R} H^{n}$.

\section{Proof of Theorem 2}

Let $M$ be a real hypersurface in $M_{n}(c), n \geq 3$, with $\eta$-recurrent shape operator, i.e.,

$$
\left\langle\left(\nabla_{X} A\right) Y, Z\right\rangle=\omega(X)\langle A Y, Z\rangle
$$

for any $X, Y, Z \in \Gamma(\mathcal{D})$, where $\omega$ is a 1 -form on $M$. By virtue of Theorem 1, we only need to consider the non-Hopf case. In this case, $\beta:=\|\phi A \xi\|>0$ and we may define a unit vector field $U:=-\beta^{-1} \phi^{2} A \xi$.

It suffices to prove that $A$ is $\eta$-parallel or $\omega=0$ according to Theorem 4. Suppose to the contrary that $\omega \neq 0$. Let $W^{\prime}$ be the vector field dual to $\omega$ and $b:=\left\|\phi W^{\prime}\right\|$. Then $b>0$ at some open subset $G$ of $M$. Since we only study local geometric property, we may identify $M$ with this open subset $G$ and define a unit vector field $W=-b^{-1} \phi^{2} W^{\prime}$. Hence (3) can be rewritten as

$$
\left\langle\left(\nabla_{X} A\right) Y, Z\right\rangle=b\langle X, W\rangle\langle A Y, Z\rangle
$$

for any $X, Y, Z \in \Gamma(\mathcal{D})$. It follow from the Codazzi equation, (4) and the fact $b>0$ that

$$
\langle X, W\rangle\langle A Y, Z\rangle=\langle Y, W\rangle\langle A X, Z\rangle .
$$

By putting $X=Z=W$ in the above equation, we obtain $\phi A W=\gamma \phi W$, where $\gamma=$ $\langle A W, W\rangle$. Hence, after putting $X=W$ in the above equation, we have

$$
\langle A Y, Z\rangle=\gamma\langle Y, W\rangle\langle Z, W\rangle
$$

for any $Y, Z \in \Gamma(\mathcal{D})$.

By (4) and (5), we see that $\gamma=0$ is equivalent to $\omega=0$. Hence, we get $\gamma \neq 0$. By differentiating covariantly both sides of the above equation in the direction of $X \in \Gamma(\mathcal{D})$; with the help of (1), (2) and 5, we have 


$$
\begin{aligned}
& \left\langle\left(\nabla_{X} A\right) Y, Z\right\rangle-\beta\langle Y, \phi A X\rangle\langle U, Z\rangle-\beta\langle Z, \phi A X\rangle\langle Y, U\rangle \\
& \quad=d \gamma(X)\langle Y, W\rangle\langle Z, W\rangle+\gamma\left\langle Y, \nabla_{X} W\right\rangle\langle Z, W\rangle+\gamma\langle Y, W\rangle\left\langle Z, \nabla_{X} W\right\rangle .
\end{aligned}
$$

By using (4) and (5), the above equation becomes

$$
\begin{gathered}
\gamma b\langle X, W\rangle\langle Y, W\rangle\langle Z, W\rangle-\gamma \beta\langle Y, \phi W\rangle\langle X, W\rangle\langle U, Z\rangle-\gamma \beta\langle Z, \phi W\rangle\langle X, W\rangle\langle Y, U\rangle \\
=d \gamma(X)\langle Y, W\rangle\langle Z, W\rangle+\gamma\left\langle Y, \nabla_{X} W\right\rangle\langle Z, W\rangle+\gamma\langle Y, W\rangle\left\langle Z, \nabla_{X} W\right\rangle .
\end{gathered}
$$

If we let $Y=Z=W$ in the above equation, then $\gamma b\langle X, W\rangle=d \gamma(X)$, for any $X \in \Gamma(\mathcal{D})$. With this fact, (7) reduces to

$$
\begin{gathered}
-\beta\langle Y, \phi W\rangle\langle X, W\rangle\langle U, Z\rangle-\beta\langle Z, \phi W\rangle\langle X, W\rangle\langle Y, U\rangle \\
=\left\langle Y, \nabla_{X} W\right\rangle\langle Z, W\rangle+\langle Y, W\rangle\left\langle Z, \nabla_{X} W\right\rangle .
\end{gathered}
$$

Next, by letting $X=W, Y=Z=\phi W$ in (8), we have $\langle\phi W, U\rangle=0$. Finally, after putting $X=W$ and $Z=\phi W$ in (8), yields $-\beta U=\left\langle\phi W, \nabla_{W} W\right\rangle W$. Since both $U$ and $W$ are unit vector fields, we may, without loss of generality, assume that $U=W$. This, together with (5), yields $A U=\beta \xi+\gamma U$ and $A Z=0$, for any $Z \perp U, \xi$. According to Lemma 3, we can see that $\gamma=0$. This contradicts the fact that $\gamma \neq 0$ and so the proof is completed.

The following result has been obtained in [7].

THEOREM 5 ([7]). Let $M$ be a real hypersurface in $M_{n}(c), n \geq 3, c \neq 0$. Then $M$ satisfies

$$
\begin{gathered}
\left(\nabla_{X} A\right) Y=\{-c\langle\phi X, Y\rangle+\eta(A Y)\langle X, \phi A \xi\rangle+\eta(A X)\langle Y, \phi A \xi\rangle \\
+\varepsilon\langle(\phi A-A \phi) X, Y\rangle\} \xi
\end{gathered}
$$

for any $X, Y \in \Gamma(\mathcal{D})$, where $\varepsilon$ is a constant, if and only if $M$ is locally congruent to one of the spaces stated in Theorem 2.

By Theorem 2 and Theorem 5, we can characterize the $\eta$-recurrence of $A$ by an expression of the covariant derivative of $A$ on the holomorphic distribution.

COROLlary 6. Let $M$ be a real hypersurface in $M_{n}(c), n \geq 3, c \neq 0$. Then the following are equivalent:

1. the shape operator $A$ is $\eta$-recurrent;

2. $\left(\nabla_{X} A\right) Y=\{-c\langle\phi X, Y\rangle+\eta(A Y)\langle X, \phi A \xi\rangle+\eta(A X)\langle Y, \phi A \xi\rangle+\varepsilon\langle(\phi A-A \phi) X, Y\rangle\} \xi$, for any $X, Y \in \Gamma(\mathcal{D})$, where $\varepsilon$ is a constant;

3. $M$ is locally congruent to one of the spaces stated in Theorem 2.

ACKNOWLEDGEMENT. The author is thankful to the referee for several valuable comments towards the improvement of the present paper. 


\section{References}

[1] J. BERNDT, Real hypersurfaces with constant principal curvatures in complex hyperbolic space, J. Reine Angew. Math. 395, 132-141 (1989).

[ 2 ] T. E. CECIL and P. J. RYAN, Focal sets and real hypersurfaces in complex projective space, Trans. Am. Math. Soc. 269, 481-499 (1982).

[ 3 ] T. HAMADA, On real hypersurfaces of a complex projective space with recurrent second fundamental form, J. Ramanujan Math. Soc. 11, 103-107 (1996).

[ 4 ] T. HAMADA, On real hypersurfaces of a complex projective space with $\eta$-recurrent second fundamental tensor, Nihonkai Math. J. 6, 153-163 (1995).

[ 5 ] M. Kimura, Real hypersurfaces and complex submanifolds in complex projective space, Trans. Am. Math. Soc. 296, 137-149 (1986).

[6] M. Kimura and S. MAedA, On real hypersurfaces of a complex projective space, Math. Z. 202, 299-311 (1989).

[ 7 ] S. H. Kon and T. H. Loo, On characterizations of real hypersurfaces in a complex space form with $\eta$-parallel shape operator, Can. Math. Bull. 55, 114-126 (2012).

[ 8 ] S. H. Kon and T. H. Loo, Real hypersurfaces in a complex space form with $\eta$-parallel shape operator, Math. Z. 269, 47-58 (2011).

[9] S. H. Kon, T. H. Loo and S. REN, Real hypersurfaces in a complex space form with a condition on the structure Jacobi operator, Math. Slovaca 64, 1007-1018 (2014).

[10] M. LohnherR and H. ReckZIEgel, On ruled real hypersurfaces in complex space forms, Geom. Dedic. 74, 267-286 (1999).

[11] S. M. LYU and Y. J. SUH, Real hypersurfaces in complex hyperbolic space with $\eta$-recurrent second fundamental tensor, Nihonkai Math. J. 8, 19-27 (1997).

[12] S. MontiEL, Real hypersurfaces of a complex hyperbolic space, J. Math. Soc. Jpn. 37, 515-535 (1985).

[13] Y. J. SUH, On real hypersurfaces of a complex space forms with $\eta$-parallel Ricci tensor, Tsukuba J. Math. 14, 27-37 (1990).

[14] R. TAKAGI, On homogeneous real hypersurfaces in a complex projective space, Osaka J. Math. 10, 495-506 (1973).

Present Address:

InSTITUTE of MATHEMATICAL SCIENCES,

UNIVERSITY OF MALAYA,

50603 KuAla Lumpur, Malaysia.

e-mail: looth@um.edu.my 